\title{
SOME PROPERTIES OF LIMITED CONTINUA, IRREDUCIBLE BETWEEN TWO POINTS*
}

\author{
BY \\ WALLACE ALVIN WILSON
}

1. In reading papers on the theory of point aggregates one frequently meets with the continuum $C$ whose points are defined as follows: when $-1 \leqq x<0$ or $0<x \leqq 1, y=\sin (1 / x)$; when $x=0,-1 \leqq y \leqq 1$. This is perhaps the most commonly cited example of a limited continuum irreducible between two points and having a continuum of condensation. If we regard the concept of connectedness im kleinen as the analogue for continua of continuity in functions, we notice that the properties of this continuum resemble those of pointwise discontinuous functions. The points where the continuum is connected im kleinen form a set of the secondary category with respect to $C$, while those of the second genre $\dagger$ form a set of the first category.

The question at once arises as to whether this similarity is of a general character or is merely due to the nature of the example cited, and suggests that a study of limited continua irreducible between two points with special reference to the oscillation at the various points would be of interest. This problem has been discussed in a paper by H. Hahn, $\ddagger$ who has shown that such a continuum is the sum of a set of sub-continua known as "prime parts," no two of which have common points. However, the fact that in many cases a prime part itself can be subdivided indicates that the subject has not been exhausted and it is the purpose of this paper to present some further results along this line.

The first half of the paper $(\$ \S 4-15)$ is devoted to the general properties of the oscillation of a limited irreducible continuum. In the second half the properties such continua have when the points of the first genre are everywhere dense are treated, and in particular it is shown that in this case there is a correspondence between the points of such a continuum and those of a linear segment analogous to that between the variables $y$ and $x$ when $y=f(x)$ is a pointwise discontinuous function of a certain type.

* Presented to the Society, September 10, 1925; received by the editors in October, 1925.

† A point of a continuum is of the first or second genre according as the oscillation of the continuum at the point is zero or not. See S. Mazurkiewicz, Sur les lignes de Jordan, F u nda menta Mathematicae, vol. 1, p. 170.

$\ddagger$ H. Hahn, Über irreduzible Kontinua, W i e ner Sitzungsberichte, vol. 130, pp. 217-250. 
2. In a previous paper, ${ }^{*}$ of which this is in one sense a continuation, two concepts were introduced which will be used throughout this work. The first was an auxiliary function $\tau(x)$, suggested as a definition of oscillation alternative to that of Mazurkiewicz. $\dagger$ This is defined as follows. Let $c$ be a point of the continuum $A$, let $V(c)$ denote those points of $A$ whose distance from $c$ is less than $\delta$ and let $C_{\delta}$ be any sub-continuum of $A$ containing $V(c)$. Then the lower bound of the diameters of all such sub-continua $C_{\delta}$ for all values of $\delta>0$ is denoted by $\tau(c)$. The function $\tau(c)$ will be called the oscillation of $A$ at $c$.

The second concept was that of oscillatory sets. Let $\delta_{1}>\delta_{2}>\cdots$, $\delta_{i} \rightarrow 0$, and let $\left\{C_{\delta_{i}}\right\}$ be a monotone decreasing sequence of sub-continua of $A$ irreducible about $V_{\delta_{i}}(c)$. Then $C(c)=D v\left[C_{\delta_{i}}\right]$ is called an oscillatory set of $A$ about $c$. It is obvious that, if $A$ is limited, each oscillatory set is a continuum.

Among the general properties of these concepts derived in the paper referred to, the following will be used.

(a) For limited continua there exist one or more oscillatory sets at each point and $\tau(c)$ is the lower bound of the diameters of the oscillatory sets of $A$ about $c$.

(b) If $\tau(c)=0$, the point $c$ is one of the oscillatory sets of $A$ about $c$.

(c) The function $\tau(c)$ is related to Mazurkiewicz' oscillatory function $\sigma(c)$ by the inequalities $\sigma(c) \leqq \tau(c) \leqq 2 \sigma(c)$.

3. Notation. The ordinary notation of the theory of aggregates is employed, with the following modifications.

The notation $A \subset B$ means that $A$ is a real part of $B$. If $A$ is a part of $B$ and may be identical with $B$, we write $A \subseteq B$.

If $A$ is the common part of the system of aggregates $\{C\}$, we write $A=D v[C]$.

If $A$ contains every element of each of the system of aggregates $\{C\}$ and no other elements, we write $A=U[C]$.

When the notation $A=B+C$ is used, it will be understood unless expressly stated to the contrary that $B \cdot C=0$.

The phrase "irreducible continuum $a b$ " means a continuum which is irreducible between the points $a$ and $b$.

The symbol $S_{\delta}(c)$ denotes an open sphere (or hypersphere) of center $c$ and radius $\delta$. If $c$ is a point of the aggregate $A$, the symbol $V_{\delta}(c)$ denotes the the subset of points of $A$ each of which has a distance from $c$ less than $\delta$.

*W. A. Wilson, On the oscillation of a continuum, these Transactions,vol. 27, pp. 429-440.

† Loc. cit. 
Then $V_{\delta}(c)=A \cdot S_{\delta}(c)$ and $V(c)$ is a region relative to $A$. In like manner, if $E$ is a closed subset of $A$, the symbol $V_{\delta}(E)$ denotes the subset of points of $A$ each of which has a distance from $E$ less than $\delta$.

4. In the following chain of six theorems, of which the first three are essentially lemmas, certain general properties of a limited irreducible continuum $a b$ are developed. It is shown that $a b$ has about each point a unique oscillatory set of diameter $\tau(c)$, that at no point $x$ of an oscillatory set of diameter greater than zero is $\tau(x)=0$, and that $\tau(c)$ is identical with Mazurkiewicz' oscillatory function $\sigma(c)$.

Theorem. Let $a b$ be a limited irreducible continuum. Let $A$ and $B$ be true sub-continua of $a b$ containing $a$ and $b$ respectively and let $A \cdot B=0$. Then there is one and only one sub-continuum $K$ of $a b$ which is irreducible between $A$ and $B$, and $K=\overline{a b-(A+B)}$.

Proof. By a continuum $K$ irreducible between $A$ and $B$ we mean a continuum containing points of both $A$ and $B$ such that no true sub-continuum contains points of both these sets. Let $L=a b-(A+B)$ and consider $\bar{L}$. Since $a b$ is a continuum,

$$
a b=A+\bar{L}+B, \quad A \cdot \bar{L} \neq 0, \quad \bar{L} \cdot B \neq 0 .
$$

Now there is a partition of $L$ into two closed sets $C$ and $D$ such that $C \cdot D=C \cdot B=D \cdot A=0, A \cdot C \neq 0$, and $B \cdot D \neq 0$; or there is not. In the former case, $a b=(A+C)+(B+D)$ and $(A+C)(B+D)=0$, which is contrary to the fact that $a b$ is a continuum.

In the latter case an easy generalization of two theorems of Janiszewski* shows that $\bar{L}$ contains a continuum $K$ joining $A$ and $B$. Since $a b$ is irreducible, $K \supseteq a b-(A+B)=L$, whence $K \supseteq \bar{L}$. Thus $\bar{L}$ is a continuum joining $A$ and $B$. Finally, any sub-continuum of $a b$ irreducible between $A$ and $B$ must contain $L$ and consequently $\bar{L}$, since $a b$ is irreducible. Hence the theorem. $\dagger$

Corollary. Let ab be a limited irreducible continuum and $A$ be a true sub-continuum containing $a$ but not $b$. Then there is one and only one sub-continuum $K$ of $a b$ which is irreducible between $A$ and $b$, and $K=\overline{a b-A}$.

5. Theorem. Let $C$ be a limited continuum. Let $a$ and $c$ be points of $C$ and let $\delta<\operatorname{Dist}(a, c)$. Let $A$ be the saturated sub-continuum of $C$ containing $a$, but no point of $V_{\delta}(c)$. Then at least one point of $A$ is a limiting point of $V_{\delta}(c)$.

- Z. Janiszewski, Sur les continus irreductibles entre deux points, Jo u rnal de l'E cole Polytechnique, ser. 2, vol. 16 (1912), pp. 109-111.

+ The proof here given was suggested by the referee in lieu of a longer one in the paper as originally submitted. 
Proof. Let $S_{\delta}$ denote the interior of a sphere of center $c$ and radius $\delta$. Let $Z$ denote the whole of the $n$-way euclidean space containing $C$, and $G_{\delta}$ be the complement of $S_{\delta}$. Then $G_{\delta}$ is closed and

$$
Z=G_{\delta}+S_{\delta}
$$

Let $\sigma<\delta$ and let $V$ denote those points of $Z$ whose distance from the closed set $A$ is not greater than $\sigma$. Then $V$ is closed and does not contain $c$. Also

$$
V=V \cdot G_{8}+V \cdot S_{8} .
$$

By a well known theorem of Janiszewski* there is a sub-continuum $K$ of $C$ joining a point $z$ of $A$ to a point $y$ of Front $V$ and lying wholly in $V$. If $K \subseteq V \cdot G_{\delta}$ then $K$ would contain no point of $S_{\delta}$, while $K \cdot A \neq 0$. This would make $K \subseteq A$ contrary to the fact that $y$ is not a point of $A$. Thus $K$ contains at least one point in $V \cdot S_{\delta}$. But $K \subset V$. Hence at least one point of $C$ in $S_{\delta}$ has a distance from $A$ not greater than $\sigma$.

As this is true for every $\sigma$, we have a sequence of points of $V_{\delta}(c)=C \cdot S_{\delta}$ whose distances from $A$ converge to zero. These therefore have at least one limiting point on $A$.

6. Theorem. Let $a b$ be a limited irreducible continuum and $c$ be a point of $a b$. Then there is a unique sub-continuum of ab irreducible about any $V_{8}(c)$.

Proof. Let $C_{\delta}$ be any sub-continuum of $a b$ irreducible about $V_{\delta}(c)$. There are several cases to consider.

I. If $V_{\delta}(c)$ contains both $a$ and $b, C_{\delta}$ obviously equals $a b$.

II. If $V_{\delta}(c)$ contains $b$ but not $a$, let $A$ be the saturated sub-continuum of $a b$ containing $a$ but no point of $V_{\delta}(c)$ and let $\bar{L}=\overline{a b-A}$.

Since $C_{\delta} \supseteq V_{\delta}(c), C_{\delta} \supseteq \bar{V}_{\delta}(c)$. Then, by $\S 5, A \cdot C_{\delta} \neq 0$. As $C_{\delta}$ contains $b$ and a point of $A$, we have by $\S 4$, Corollary,

$$
C_{8} \supseteq \bar{L} .
$$

But

$$
\bar{L} \supset a b-A \supseteq V_{8}(c) .
$$

Since $C_{\delta}$ is irreducible about $V_{\delta}(c)$, relations (1) and (2) give

$$
C_{\delta}=\bar{L}
$$

- See reference under $\$ 4$, p. 100. 
III. If $V_{\delta}(c)$ contains $a$ but not $b$, we have essentially the same situation as in Case II.

IV. If $V_{\delta}(c)$ contains neither $a$ nor $b$, let $A$ be defined as in Case II and let $B$ have the same relation to the point $b$, while $\bar{L}=\overline{a b-(A+B)}$. As in Case II, $A \cdot C_{\delta} \neq 0$ and $B \cdot C_{\delta} \neq 0$. Hence, by $\$ 4$,

$$
C_{\delta} \supseteq \bar{L} \text {. }
$$

But

$$
\bar{L} \supset a b-(A+B) \supseteq V_{8}(c) .
$$

Then relations (3) and (4) give

$$
C_{\S}=\bar{L} \text {. }
$$

7. THEOREM. Let ab be a limited irreducible continuum and let $c$ be a point of $a b$. Then the oscillatory set of $a b$ about $c$ is unique and its diameter is $\tau(c)$, and, if $\tau(c)=0$, it is the point $c$ itself.

Proof. Let $0<\delta<\eta$, and let $C_{\delta}$ and $C_{\eta}$ be the sub-continua of $a b$ irreducible about $V_{\delta}(c)$ and $V_{\eta}(c)$ respectively. Obviously $V_{\delta}(c) \subseteq V_{\eta}(c)$ and hence $V_{\delta}(c) \subseteq C_{\eta}$.

Then $C_{\eta}$ has a sub-continuum $D$ irreducible about $V_{\delta}(c)$. Since $D \subset C_{\eta}$,

$$
D \subseteq a b \text {. }
$$

But $C_{\delta}$ is the only sub-continuum of $a b$ irreducible about $V_{\delta}(c)$. Hence (1) gives $C_{\delta}=D$ and $C_{\delta} \subseteq C_{\eta}$.

This result shows that if $\left\{\delta_{i}\right\}$ and $\left\{\eta_{i}\right\}$ are monotone decreasing sequences converging to zero, $D v\left[C_{\delta_{i}}\right]=D v\left[C_{\eta_{i}}\right]$. Hence the oscillatory set is unique. That its diameter is $\tau(c)$ follows from the fact that for continua in general $\tau(c)$ is equal to the minimum diameter of all the oscillatory sets about $c$. (See $\S 2$.) Then if $\tau(c)=0$, the oscillatory set can contain no other point than $c$ itself.

For continua in general the oscillatory sets are not unique, as is shown in an example given in the paper referred to in $\$ 2$. The reader should note the analogy of the oscillatory set to the aggregate of limiting values of a one-valued function of a real variable $f(x)$ as $x \rightarrow a$. It is to the above theorem that the oscillatory sets of a limited irreducible continuum owe most of their value. A point of the second genre lies on a continuum of condensation and in simple cases there is a greatest such continuum, but this is not always the case. On the other hand the oscillatory set of each point is unique. 
8. THEOREM. Let ab be a limited irreducible continuum. Then for each point $c$ of $a b, \tau(c)=\sigma(c)$.

Proof. It has been shown elsewhere (see §2) that

$$
\sigma(c) \leqq \tau(c) .
$$

Let $c \neq a, c \neq b$. Then by definition* of $\sigma(c)$ any two points $x$ and $y$ of $a b$ lying in a closed sphere $\bar{S}_{\delta}(c)$ whose center is $c$ and whose radius $\delta$ is sufficiently small can be joined by a sub-continuum $C(x, y)$ of $a b$ such that

$$
\operatorname{Diam} C(x, y)<\sigma(c)+\epsilon,
$$

where $\epsilon$ is any positive quantity.

Now let $S_{\delta}(c)$ denote the interior of $\bar{S}_{\delta}(c)$, and let $\delta$ be so small that $\bar{S}_{\delta}(c)$ contains neither $a$ nor $b$. Let $A$ and $B$ be the saturated sub-continua of $a b$ containing $a$ and $b$ respectively, but no point of $V_{\delta}(c)=a b \cdot S_{\delta}(c)$. By $\S 5, A$ contains a point $x$, and $B$ a point $y$, on Front $\bar{S}_{\delta}(c)$, and these points satisfy relation (2). Then

$$
a b=A+C(x, y)+B, \quad A \cdot C(x, y) \neq 0, \quad B \cdot C(x, y) \neq 0 .
$$

Since $A \cdot V_{\delta}(c)=B \cdot V_{\delta}(c)=0$, we have

Then relation (2) gives

$$
V_{\delta}(c) \subseteq C(x, y) .
$$

$$
\tau(c) \leqq \sigma(c)+\epsilon .
$$

This with relation (1) gives the theorem for the case under consideration. Similar reasoning applies to the cases that $c=a$ or $c=b$.

9. Theorem. Let ab be a limited irreducible continuum. Let $c$ be a point of the second genre of $a b$ and let $C(c)$ be the oscillatory set of $a b$ about $c$. Then no point of $C(c)$ is of the first genre.

Proof. Assume that there is a point $x$ of $C(c)$ at which $\tau(x)=0$, and let $\eta=$ Dist $(x, c)$. Then for any positive $\epsilon<\eta$ there is a $\delta>0$, such that there is a sub-continuum $D$ of $a b$ having the property

$$
V_{\delta}(x) \subseteq D \subseteq V_{\mathrm{c}}(x) .
$$

Let $c$ be different from $a$ and $b$ and let $\delta$ be so small that $V_{\delta}(x)$ contains neither $a$ nor $b$. Let $A$ and $B$ be the saturated sub-continua of $a b$ containing $a$ and $b$ respectively, but no point of $V_{\delta}(x)$. Then by $\$ 5$ and relation (1)

$$
a b=A+D+B, \quad A \cdot D \neq 0, \quad B \cdot D \neq 0 .
$$

* See S. Mazurkiewicz, Sur les lignes de Jordan, Fundamenta Mathematicae, vol. 1, p. 170. 
Since $\epsilon<\eta, c$ is not a point of $D$; suppose that $c$ is a point of $A$. Then $A$ has a sub-continuum irreducible about any $V_{\sigma}(c)$, if $\sigma$ is so small that $V_{\sigma}(c)$ contains no point of $D$ or of $B$. Since $A \subset a b$, this is the sub-continuum of $a b$ irreducible about $V_{\sigma}(c)$ by $\S 6$. Hence we have $C(c) \subseteq A$. This is a contradiction, since $x$ is not a point of $A$.

The cases where $c$ coincides with $a$ or $b$ are treated in a like manner.

Remark. The above theorem shows that the oscillatory set about a point $c$ is a part of the prime part containing $c$. That it need not be identical with the prime part is seen from the example suggested in $\S 23$.

10. It would be natural to surmise from $\S 9$ that, if $C(c)$ is the oscillatory set of $a b$ about $c$, then $C(c)$ is the oscillatory set about each point $z$ on $C(c)$ and that $\tau(z)=\tau(c)$. This, however, is not true, as the following example shows. Let $a b$ be the continuum whose points are defined as follows: for $x=0,-1 \leqq y \leqq 1$; for $0<x \leqq 1, y=\sin ^{2}(1 / x)$; for $-1 \leqq x<0, y=-\sin ^{2}(1 / x)$. The oscillatory set of $a b$ about the point $(0,0)$ is the segment of the $y$-axis between $(0,-1)$ and $(0,1)$ and the oscillation is 2 . For the point $\left(0, \frac{1}{2}\right)$ the oscillatory set is the segment joining $(0,0)$ and $(0,1)$ and the oscillation is 1.

In order to investigate this question more fully it is necessary to consider the point set remaining when the oscillatory set about the point $c$ is subtracted from the continuum $a b$. The inference that the remainder is two semi-continua, one containing $a$ and the other $b$ (or one semi-continuum if $C(c)$ contains either $a$ or $b)$ is seen to be false for the point $\left(0, \frac{1}{2}\right)$ in the example just given. The difficulty is that $\left(0, \frac{1}{2}\right)$ is a limiting point of only one of the semi-continua containing the end points of $a b$. The inference is borne out for $(0,0)$, however, as this point is a limiting point of both semi-continua.

The following notation will now be used. If $x$ is a point of $a b$, the oscillatory set of $a b$ about $x$ will be denoted by $X(x)$, or simply $X$. The saturated semi-continua of $a b-X$ containing $a$ and $b$ respectively will be denoted by $X_{a}$ and $X_{b}$. If $b$ is a point of $X$, obviously $X_{b}=0$; if $a$ is a point of $X, X_{a}=0$. It is evident that

$$
X_{a}+X+X_{b} \subseteq a b \text { and } X_{a} \cdot X_{b}=0 .
$$

By a theorem of Janiszewski* we know that $X \cdot \bar{X}_{a} \neq 0$ and $X \cdot \bar{X}_{b} \neq 0$; hence

$$
a b=\bar{X}_{a}+X+\bar{X}_{b}, \quad X \cdot \bar{X}_{a} \neq 0, \quad X \cdot \bar{X}_{b} \neq 0 .
$$

Thus $\bar{X}_{a}+X$ and $X+\bar{X}_{b}$ are continua. Analogous theorems hold, of course, for the cases that $X_{a}=0$ and $X_{b}=0$.

* See reference under $\$ 4$, p. 123. 
It is also easy to show that

$$
\bar{X}_{a} \cdot X_{b}=0 \text { and } X_{a} \cdot \bar{X}_{b}=0 .
$$

For, let $A$ and $B$ be the saturated sub-continua of $a b$ containing $a$ and $b$ but no points of $V_{\delta}(x)$. Then $A \cdot X_{b}=0$ and $B \cdot X_{a}=0$, since $a b$ is irreducible. But

$$
a b=A+X_{\delta}(x)+B, \quad A \cdot X_{\delta}(x) \neq 0, \quad B \cdot X_{\delta}(x) \neq 0 .
$$

Then

and

$$
X_{a} \subseteq A+X_{\delta}(x)
$$

Hence

$$
\bar{X}_{a} \cdot X_{b} \subseteq A \cdot X_{b}+X_{b} \cdot X_{\delta}(x)=X_{b} \cdot X_{\delta}(x)
$$

$$
\bar{X}_{a} \cdot X_{b} \subseteq D v\left[X_{b} \cdot X_{b}(x)\right]=X \cdot X_{b}=0 .
$$

11. Theorem. Let $C$ be the oscillatory set of the limited irreducible continuum $a b$ about one of its points $c$ and let $C$ contain neither $a$ nor $b$. Let $c$ be a common limiting point of $C_{a}$ and $C_{b}$. Then $a b=C_{a}+C+C_{b}$.

Proof. Owing to the hypothesis regarding $c$ any sub-continuum $C_{\delta}(c)$ of $a b$ irreducible about $V_{\delta}(c)$ contains points of $C_{a}$ and $C_{b}$. Hence

$$
a b=C_{a}+C_{\delta}(c)+C_{b}, \quad C_{a} \cdot C_{\delta}(c) \neq 0, \quad C_{b} \cdot C_{\delta}(c) \neq 0,
$$

or

Therefore

$$
C_{8}(c) \supseteq a b-\left(C_{a}+C_{b}\right) .
$$

$$
C \supseteq a b-\left(C_{a}+C_{b}\right) \text {. }
$$

But

whence

$$
C_{a}+C+C_{b} \subseteq a b, \quad C \cdot C_{a}=0, \quad C \cdot C_{b}=0,
$$

$$
C \subseteq a b-\left(C_{a}+C_{b}\right) \text {. }
$$

Relations (1) and (2) give the theorem.

CoRollary 1. Let $C$ be the oscillatory set of the limited irreducible continuum $a b$ about the point $c$. Let $a$ be $a$ point of $C$ and $c$ be a limiting point of $C_{b}$. Then $a b=C+C_{b}$.

CoRollary 2. Under the conditions of the above theorem the sets $C_{a}+C$ and $C+C_{b}$ are continua.

For $\bar{C}_{a} C_{b}=0$ by $\S 10$, relation (3). 
Corollary 3. If $\tau(c)=0, a b=C_{a}+c+C_{b}=\bar{C}_{a}+C_{b}=C_{a}+\bar{C}_{b}$.

For by $\S 10$, relation (2), $c$ is a common limiting point of $C_{a}$ and $C_{b}$.

COROLLARY 4. Under the conditions of the above theorem, $C$ is a continuum of condensation of $a b$.

For $c$ is a point of $\bar{C}_{a} \cdot \bar{C}_{b}$. Hence $a b=\bar{C}_{a}+\bar{C}_{b}$. Therefore $C \subset \overline{C_{a}+C_{b}}$.

Analogous theorems hold for the oscillatory sets of the points $a$ and $b$.

12. From $\S 11$, Corollary 3 , we can easily deduce the following well known properties of irreducible continua.

(a) If $c$ is a point of the first genre of the limited irreducible continuum $a b$, then $c$ divides $a b$ into two unique irreducible continua $a c$ and $c b$, and $a c \cdot c b=c$.

(b) If $x$ and $y$ are two points of the first genre of the limited irreducible continuum $a b$, then $x$ and $y$ divide $a b$ into three unique irreducible continua; if $x$ is a point of $a y, a b=a x+x y+y b$, and $a x \cdot x y=x, x y \cdot y b=y$, and $a x \cdot y b=0$.

(c) If $c$ is a point of the first genre of the limited irreducible continuum $a b$ and $x$ is a point of $a c$, then every irreducible sub-continuum $a x$ of $a b$ is a real part of $a c$ and every $x b$ contains $c b$ as a real part.

It is also evident that analogous theorems can be deduced from the partition $a b=C_{a}+C+C_{b}$ of $\S 11$. These we shall not go into, but we need the two theorems immediately following.

13. Theorem. Let $X$ be the oscillatory set of the limited irreducible continuum $a b$ about the point $x$ and let $X$ contain neither $a$ nor $b$. Let $c$ be a common limiting point of $X_{a}$ and $X_{b}$. Then the oscillatory set $C$ about contains $X$.

Proof. Any sub-continuum $C_{\delta}(c)$ of $a b$ irreducible about $V_{\delta}(c)$ contains points of both $X_{a}$ and $X_{b}$. Hence

$$
C_{\delta}(c) \supseteq a b-\left(X_{a}+X_{b}\right) .
$$

But

or

$$
X_{a}+X+X_{b} \subset a b
$$

$$
X \subseteq a b-\left(X_{a}+X_{b}\right)
$$

Relations (1) and (2) give

Hence

$$
C_{\delta}(c) \supseteq X
$$

$$
C \supseteq X
$$

Analogous theorems hold for the oscillatory sets of $a$ and $b$. 
14. Theorem. Let ab be a limited irreducible continuum. Let $c$ be a point of $a b$ and let $C$ be the oscillatory set of $a b$ about $c$. Let $c$ be a common limiting point of the saturated semi-continua $C_{a}$ and $C_{b}$ of $a b-C$. Let $x$ be any point of $C$ and $X$ be the oscillatory set of $a b$ about $x$. Then $X \subseteq C$.

Proof. Let $\left\{\delta_{i}\right\}$ and $\left\{\delta_{k}\right\}$ be two decreasing sequences of positive numbers converging to zero. Let $C_{i}$ be the sub-continuum of $a b$ irreducible about $V_{\delta_{i}}(c)$, while $D_{k}$ has the corresponding meaning for $V_{\delta_{k}}(x)$. Assume that $C$ contains neither $a$ nor $b$.

Since $c$ is a limiting point of both $C_{a}$ and $C_{b}$, for every $i$ there is a subcontinuum $A_{i}$ of $C_{a}$ joining $a$ to a point of $V_{\delta_{i}}(c)$, and likewise a $B_{i}$ of analogous properties. Hence

$$
a b=A_{i}+C_{i}+B_{i}, \quad A_{i} \cdot C_{i} \neq 0, \quad C_{i} \cdot B_{i} \neq 0 .
$$

Since $C \cdot A_{i}=C \cdot B_{i}=0, V_{\delta_{k}}(x)$ contains no point of either $A_{i}$ or $B_{i}$ if $k$ is sufficiently great. Thus $V_{\delta_{k}}(x) \subseteq C_{i}$ and so for every $i$ there is a $k$ such that

$$
D_{k} \subseteq C_{i} \text {. }
$$

Since $X=D v\left[D_{k}\right]$ and $C=D v\left[C_{i}\right]$, this gives at once

$$
X \subseteq C \text {. }
$$

The special cases where $C$ contains $a$ or $b$ are treated in like manner.

Corollary. If in the above theorem $x$ is a comnion limiting point of $C_{a}$ and $C_{b}$, then the oscillatory set $X$ of ab about $x$ is identical with $C$.

15. The example of $\S 10$ shows that if a point $x$ lies on an oscillatory set $C(c)$, the oscillatory set $X(x)$ may form only a part of $C(c)$. In the example mentioned the point $\left(0, \frac{1}{2}\right)$ is an instance of this. This fact, coupled with $\S 14$, suggests setting apart as a separate class the oscillatory sets satisfying the hypotheses of that theorem. Accordingly we have the definition

If $C(c)$ is the oscillatory set of the limited irreducible continuum ab about a point $c$, we shall call $C(c)$ complete when it contains neither $a$ nor $b$ and $c$ is a common limiting point of the saturated semi-continua $C_{a}$ and $C_{b}$ of $a b-C(c)$. Likewise, if $c$ is identical with $a$ or $b$ and $c$ is a limiting point of $C_{b}$ or $C_{a}$, respectively, we shall call $C(c)$ complete.

In addition to the properties already derived in $\$ \S 11-14$, we have the following general theorem.

Let $a b$ be a limited irreducible continuum and the oscillatory set $C(c)$ be complete. Let $x$ be a point of $C_{a}$ or $C_{b}$. Then the oscillatory set $X(x)$ is a part of $C_{a}$ or $C_{b}$, respectively. 
Proof. To fix the ideas let $x$ be a point of $C_{a}$. Then it follows from the definition of oscillatory sets that there is a $\delta>0$ so small that, if $C_{\delta}$ is the sub-continuum of $a b$ irreducible about $V_{\delta}(c), C_{\delta}$ does not contain $x$. Since $C(c)$ is complete, there is a sub-continuum $E$ of $C_{a}$ containing $a$ and a point in $V_{\delta}(c)$. Then

$$
a b=E+C_{\delta}+C_{b} \text { and } E \cdot C_{b}=0, \quad E \cdot C_{\delta} \neq 0, \quad C_{\delta} \cdot C_{b} \neq 0 .
$$

Since $x$ is not a point of $C_{\delta}$ or $C_{b}$, it is a point of $E$. Then for some $\eta>0$. $V_{\eta}(x) \subset E$. Then by the definition of oscillatory set,

$$
X(x) \subseteq E \subset C_{a},
$$

which was to be proved.

A similar theorem holds when the oscillatory set of $a$ or $b$ is complete.

16. Simple irreducible continua. From $\S 11$, Corollary 3 , we see that each point of the first genre is a complete oscillatory set. We now turn to a consideration of limited continua, irreducible between two points and containing an everywhere dense set of points of the first genre. To save repetition in the statements of theorems, we shall call such continua simple irreducible continua. It may be remarked here that it is evident that the oscillatory sets of such continua about $a$ and $b$ are always complete. Furthermore, if $x^{\prime}$ and $x^{\prime \prime}$ are two points of the first genre of $a b$ and a complete oscillatory set $C$ is contained in the sub-continuum $x^{\prime} x^{\prime \prime}$, it follows from $\S 12(b)$ and $\S 11$, Corollary 4 , that $C$ is a continuum of condensation of $x^{\prime} x^{\prime \prime}$. The following lemmas are convenient in later work.

LemMa I. Let $a b$ be a simple irreducible continuum and let $C$ be a complete oscillatory set containing neither $a$ nor $b$. Then there are sequences of points $\left\{x_{i}\right\}$ and $\left\{x_{j}\right\}$ of the first genre converging to $c$ and lying in $C_{a}$ and $C_{b}$, respectively, and $C=D v\left[x_{i} x_{j}\right]$.

Proof. If $y_{i}$ is any point of $C_{a}$, there is a $\delta_{i}$ so small that $V_{\delta_{i}}\left(y_{i}\right)$ contains no point of $C$ or $C_{b}$. But every $V_{\delta}\left(y_{i}\right)$ contains points of the first genre. Hence it is evident that any sequence $\left\{y_{i}\right\}$ of points of $C_{a}$ converging to $c$ can be replaced by a sequence of points of the first genre belonging to $C_{a}$ and converging to $c$. Thus the sequences $\left\{x_{i}\right\}$ and $\left\{x_{j}\right\}$ exist.

Now for any $\eta, V_{\eta}(c)$ contains an $x_{i}$ and an $x_{j}$. Hence $C \supseteq D v\left\{x_{i} x_{j}\right\}$. On the other hand, for any given $x_{i}$ and $x_{j}$ there is an $\eta$ so small that $V_{\eta}(c) \subseteq x_{i} x_{j}$. Thus $C \subseteq x_{i} x_{j}$ and hence $C \subseteq D v\left[x_{i} x_{j}\right]$. Therefore $C=D v\left[x_{i} x_{i}\right]$.

LEMMA II. Let ab be a simple irreducible continuum and $A$ be the oscillatory set about $a$. Then there is a sequence of points $\left\{x_{i}\right\}$ of the first genre converging to $a$ and lying in $A_{b}$, and $A=D v\left[a x_{i}\right]$. 
This and the corresponding lemma for the point $b$ are proved in the same way as Lemma $\mathbf{I}$.

17. Theorem. Let ab be a simple irreducible continuum. Then (i) no two complete oscillatory sets have common points; (ii) every incomplete oscillatory set $Y$ contains a point whose oscillatory set is complete and contains $Y$; (iii) every point lies on one and only one complete oscillatory set; and (iv) if two oscillatory sets have common points, one is complete and contains the other, or both are contained in the same complete oscillatory set.

Proof. (i) Let $X$ be a complete oscillatory set and $a b=X_{a}+X+X_{b}$, where either $X_{a}$ or $X_{b}$ may be void. Let $Y=Y(y)$ be another complete oscillatory set. If $y$ is a point of $X_{a}$ or $X_{b}$, say the former, $\S 15$ shows that $Y \subset X_{a}$; hence $X \cdot Y=0$. If $y$ is a point of $X$, then $\S 14$ shows that

$$
Y \subseteq X \text {. }
$$

In this case we have $a b=Y_{a}+Y+Y_{b}$. If $x$ is not a point of $Y$, then by $\S 15 X$ is a part of $Y_{a}$ or $Y_{b}$, which is false, since $X \cdot Y \supset y$. Then $x$ is a point of $Y$ and hence

$$
X \subseteq Y
$$

Then either $X \cdot Y=0$, or by relations (1) and (2) $X=Y$.

(ii) Let $Y$ be an oscillatory set which is not complete. If $c$ is a point of the first genre, $Y \subset a c$ or $Y \subset c b$, by $\S 15$. Then $c$ is a point of $Y_{b}$ or $Y_{a}$, respectively. Thus all the points of the first genre are on $Y_{a}$ and $Y_{b}$. As they are everywhere dense in $a b, Y=Y \cdot \bar{Y}_{a}+\cdot Y \bar{Y}_{b}, Y \cdot \bar{Y}_{a} \neq 0, Y \cdot \bar{Y}_{b} \neq 0$. Since $Y$ is a continuum, at least one point $x$ of $Y$ is a limiting point of both $Y_{a}$ and $Y_{b}$. Then, by $\S 13, Y$ is a part of the oscillatory set $X$ about $x$.

To show that $X$ is complete, observe first that $X_{a} \subseteq Y_{a}$ and $X_{b} \subseteq Y_{b}$. Then, as all the points of the first genre are on $X_{a}$ and $X_{b}, X_{a}$ contains all the points of $Y_{a}$ that are of the first genre. Since by $\S 10, Y_{a} \cdot \bar{Y}_{b}=0$, for every point $z$ of $Y_{a}$ any $V_{\delta}(z)$ contains points of the first genre lying on $Y_{a}$. Then, as $x$ is a limiting point of $Y_{a}$, it is a limiting point of points of the first genre of $Y_{a}$ and consequently a limiting point of $X_{a}$. Likewise it is a limiting point of $X_{b}$. Hence $X$ is complete.

(iii) This follows from (i) and (ii).

(iv) If one of two oscillatory sets with common points is complete, it contains the other by (i) and (ii). If neither is complete, each lies in a complete oscillatory set by (ii). These complete oscillatory sets must be identical by (i). 
18. The aggregate of complete oscillatory sets. Let $X$ be any complete oscillatory set of the simple irreducible continuum $a b$, and let $K=\{X\}$ be the aggregate whose elements are these complete oscillatory sets. From $\S 17$ it follows that $a b=U[X]$ and that no two sets $X$ have common points.

The fact that $a b=X_{a}+X+X_{b}$ permits us to order the aggregate $K$. For, if $Y$ is another element of $K$, we have seen $(\$ 17)$ that either $Y \subset X_{a}$ or $Y \subset X_{b}$. In the former case we say that $Y$ precedes $X$ and write $Y<X$; in the latter, that $Y$ follows $X$ and we write $Y>X$. It is easy to show that $X>Y$ if $Y<X$ and $X<Y$ if $Y>X$, and that, if $X, Y$, and $Z$ are three complete oscillatory sets such that $X<Y$ and $Y<Z$, then $X<Z$. Thus $K$ is a simply ordered aggregate. Furthermore $K$ has a first and a last element, namely the complete oscillatory sets containing $a$ and $b$, respectively.

We shall now proceed to show that the order type of the set $K$ is similar to that of the aggregate whose elements are the points of a finite closed segment. Then by virtue of this similarity we shall determine a correspondence between the points of $a b$ and those of a linear segment.

19. THEOREM. The aggregate of complete oscillatory sets of a simple irreducible continuum ab has a dense order type.

Proof. We must prove that, if $X$ and $Y$ are two complete oscillatory sets, then there is a third lying between them. Let $X<Y$. Then

$$
a b=X_{a}+X+X_{b},
$$

and

$$
a b=Y_{a}+Y+Y_{b}
$$

Since $X<Y$ it follows that

$$
Y+Y_{b} \subseteq X_{b}
$$

If $Y+Y_{b}=X_{b}$, relation (1) gives

and

$$
a b=X_{a}+X+Y+Y_{b},
$$

$$
\left(X_{a}+X\right) \cdot\left(Y+Y_{b}\right)=0 .
$$

This is an obvious contradiction, since $a b$ is a continuum.

Hence $X_{b}$ contains at least one point $z$ not belonging to $Y+Y_{b}$. Therefore $z$ is a point of $Y_{a}$ and the complete oscillatory set $Z$ containing $z$ is a part of $Y_{a}$. Thus $Z<Y$. But, as $z$ is also a point of $X_{b}, Z \subset X_{b}$ and $Z>X$. This proves the theorem. 
20. TheOREM. Let ab be a simple irreducible continuum. Then the aggregate $K$ of complete oscillatory sets of ab has an open enumerable subset which is dense in itself and also dense in ab.

Proof. Since the set of points of the first genre is dense in $a b$, there is an enumerable subset of this which is also dense in $a b$; call it $E$. Also, if $a$ or $b$ is of the first genre, we can assume that $E$ contains neither of these.

Now let $X$ and $Y$ be two elements of $K$ and let $X<Y$. By $\S 19$ there is an element $Z$ of $K$ such that

$$
X<Z<Y \text {. }
$$

Then $Z$ contains no points of the continua $X_{a}+X$ and $Y+Y_{b}$. Then if $z$ is a point of $Z$, for $\delta$ sufficiently small

$$
V_{b}(z) \subset X_{b} \text { and } V_{b}(z) \subset Y_{a} \text {. }
$$

But $E$ is dense in $a b$; hence $V_{\delta}(z)$ contains a point $e$ of $E$. As $\tau(e)=0$, the complete oscillatory set containing $e$ is $e$ itself. Then the complete oscillatory set of $e$ is a part of both $X_{b}$ and $Y_{a}$. Hence

$$
X<e<Y \text {. }
$$

Therefore $E$ considered as an aggregate of complete oscillatory sets is dense in $K$ and obviously dense in itself. It is also evident that it has no first and no last element.

21. THEOREM. Let ab be a simple irreducible continuum, and let $K$ be the aggregate of complete oscillatory sets of $a b$. Then the order type of $K$ is that of a finite segment.

Proof. Let $K=P+Q$ be a partition such that every element of $P$ precedes every element of $Q$. Let $X$ be any element of $P$ and let $Y$ be any element of $Q$; then $P=\{X\}$ and $Q=\{Y\}$. Also $B=U[X]$ and $C=U[Y]$. Then we have

$$
a b=B+C .
$$

Suppose now that $P$ has no last and $Q$ no first element. Let $Y$ be any element of $Q$. Then there is an element $Y^{\prime}$ of $Q$ preceding $Y$ and every element $X$ of $P$ precedes $Y^{\prime}$. Then $B=U[X] \subset Y_{a}^{\prime}$ and

$$
\bar{B} \subseteq Y_{a}^{\prime}+Y^{\prime} \subseteq Y_{a} .
$$

Hence $\bar{B} \cdot Y=0$ and no point of $\bar{B}$ lies in $C=U[Y]$.

In the same way we show that $B \cdot \bar{C}=0$. Since $\bar{B} \cdot C+B \cdot \bar{C}=0$, we have a contradiction, as $a b$ is a continuum. Hence either $P$ has a last, or $Q$ has a 
first element. As it has been proved in $\S 19$ that $K$ is dense, this shows that $K$ is continuous.*

If we omit from $K$ the complete oscillatory sets of $a$ and $b$, the resulting set $K^{\prime}$ is also continuous and by $\S 20 K^{\prime}$ contains an open enumerable aggregate dense in itself and dense in $K^{\prime}$. Therefore the order type of $K^{\prime}$ is that of the linear continuum $\dagger$ and $K$ is similar to a finite closed segment.

22. The effect of the theorem just proved is to set up a uniform correspondence between the points of a finite segment $(0 \leqq t \leqq 1)$ and the complete oscillatory sets of a simple irreducible continuum $a b=\{x\}$. This defines a correspondence between the points of $a b$ and those of the segment, which need not be uniform. If we denote this by $x=f(t)$, we have a function which is in general multivalued. It is, moreover, but a slight extension of the ordinary notion of continuity if we say that $f(t)$ is continuous at a point $t^{\prime}$ when $f\left(t^{\prime}\right)$ is a single point $x^{\prime}$ and for every $\epsilon>0$, the aggregate of images of all points $t$ in some $V_{\delta}\left(t^{\prime}\right)$ lie in $V_{\epsilon}\left(x^{\prime}\right)$.

Theorem. Let $a b=\{x\}$ be a simple irreducible continuum. Then there is a correspondence $x=f(t)$ between the points of $a b$ and those of the unit segment $T=(0 \leqq t \leqq 1)$ having the following properties:

(i) To each point $t$ corresponds one and only one point of the first genre or one and only one complete oscillatory set.

(ii) $f(t)$ is continuous at each point $t$ whose image is a single point.

(iii) At a point $t_{0}$ whose image is not a single point the corresponding oscillatory set $X_{0}=\left\{x_{0}\right\}, x_{0}=f\left(t_{0}\right)$, is the set of accumulation $\ddagger$ of the sets $x=f(t)$ as $t \rightarrow t_{0}$.

(iv) The images of 0 and 1 are the complete oscillatory sets of $a$ and $b$, respectively.

Proof. The correspondence in question is that defined by the similarity of the aggregate of complete oscillatory sets $K=\{X\}$ of $a b$ to any finite closed segment. This gives us (i) and (iv) at once. It remains to prove (ii) and (iii).

Let $t_{0}$ be a point whose image is the single point $x_{0}$. Then $\tau\left(x_{0}\right)=0$ and this fact, with $\S 16$, shows that there are two points of the first genre, $x^{\prime}$ and $x^{\prime \prime}$, such that $x^{\prime}<x_{0}<x^{\prime \prime}$ and the irreducible sub-continuum $x^{\prime} x^{\prime \prime}$ is a

* See F. Hausdorff, Grundzüge der Mengenlehre, p. 90.

$\dagger$ Ibid., p. 101.

$\ddagger$ This notion is defined by Janiszewski (loc cit., p. 93) for the case of an enumerable system of sets. The following is a natural extension. Let $\{f(t)\}$ denote a system of sets depending on the parameter $t$, which ranges over an interval and let $t_{0}$ be any point in this interval. Let $F$ be the class of all points $\{x\}$ such that, for every $\epsilon>0, S_{\epsilon}(x)$ contains a point of some $f(t)$ for at least one $t$ in every $V_{\delta}\left(t_{0}\right), \delta>0$. Then $F$ is the aggregate of accumulation of the sets $f(t)$ as $t \rightarrow t_{0}$. 
part of $V_{e}\left(x_{0}\right)$ for a fixed positive $\epsilon$. Let $x^{\prime}=f\left(t^{\prime}\right)$ and $x^{\prime \prime}=f\left(t^{\prime \prime}\right)$. Then by similarity, $t^{\prime}<t_{0}<t^{\prime \prime}$. Now for $\delta$ sufficiently small $V_{\delta}\left(t_{0}\right) \subset t^{\prime} t^{\prime \prime}$. Hence the images of points in $V_{\delta}\left(t_{0}\right)$ lie in $x^{\prime} x^{\prime \prime}$, since $x^{\prime}<X<x^{\prime \prime}$, when $t^{\prime}<t<t^{\prime \prime}$. But then they lie in $V_{\epsilon}\left(x_{0}\right)$ and we have continuity. The cases where $t_{0}=0$ or $t_{0}=1$ are treated similarly.

Now let $t_{0}$ be a point for which the corresponding oscillatory set $X_{0}$ contains more than one point. Let $x_{0}$ be a point of $X_{0}$ which is a common limiting point of the saturated semi-continua $X_{a}$ and $X_{b}$ of $a b-X_{0}$. Then, by $\S 16$, $X_{a}$ and $X_{b}$ contain respectively sequences $\left\{x_{i}\right\}$ and $\left\{x_{j}\right\}$ of points of the first genre converging to $x_{0}$ and $X_{0}=D v\left[x_{i} x_{j}\right]$. Hence for any $\epsilon>0$ there is an $x^{\prime}$ and an $x^{\prime \prime}$, points of the first genre, such that

$$
x^{\prime}<X_{0}<x^{\prime \prime} \text { and } X_{0} \subset x^{\prime} x^{\prime \prime} \subset V_{\mathrm{e}}\left(X_{0}\right) \text {. }
$$

Let $x^{\prime}=f\left(t^{\prime}\right), x^{\prime \prime}=f\left(t^{\prime \prime}\right)$. Then $t^{\prime}<t_{0}<t^{\prime \prime}$. For $\delta$ sufficiently small, then, the images of all points in $V_{\delta}\left(t_{0}\right)$ will lie in $x^{\prime} x^{\prime \prime}$ and consequently in $V .\left(X_{0}\right)$. Hence, as $t \rightarrow t_{0}$, the points of accumulation of the corresponding points $x$ will be contained in $X_{0}$.

Now let $x$ be any point in $X_{0}$. Let $\epsilon>0$. For any $\delta>0$ there are points $t_{i}$ and $t_{j}$ in $V_{\delta}\left(t_{0}\right)$ such that $t_{i}<t_{0}<t_{j}$ and the images of $t_{i}$ and $t_{j}$ are points of the first genre. Let $x_{i}=f\left(t_{i}\right)$ and $x_{i}=f\left(t_{j}\right)$. By $\S 16, X_{0}$ is a continuum of condensation of $x_{i} x_{j}$; hence $V_{c}(x)$ contains at least one point $x^{\prime}$ of $x_{i} x_{i}$ which is not on $X_{0}$. But if $x^{\prime}=f\left(t^{\prime}\right)$, then $t^{\prime}$ lies in $V_{\delta}\left(t_{0}\right)$. Thus each point of $X_{0}$ is a point of accumulation of the points $x=f(t)$ as $t \rightarrow t_{0}$, by definition.

This, with the preceding paragraph, completes the proof of (iii).

23. The nature of the correspondence involved in the theorem just proved is made clearer by relating it to the function theory. If $x=f(t)$ is a limited one-valued function defined for a set $E$ of values of $t$ everywhere dense in the interval $a \leqq t \leqq b$ and continuous at each point, it is well known that in general there is no function continuous at every point of $a b$ and equal to $f(t)$ at the points of $E$, for the reason that, at a point $c$ not in $E, \lim _{t \rightarrow c} f(t)$ may not exist. If, however, we agree to set $F(t)=f(t)$ at the points of $E$ and to assign to $F(t)$ at $t=c$ the aggregate of limiting values of $f(t)$ as $t \rightarrow c$ over all possible sequences in $E$ and for each point $c$ this aggregate is a continuum, we have a multivalued function possessing many of the attributes of continuity. The graph is easily seen to be a continuum and, for any point $c$, all of the limits of $F(t)$ as $t \rightarrow c$ are values of $F(c)$. Such a function is $x=$ $\sin (1 / t)$ for $t \neq 0,-1 \leqq x \leqq 1$ for $t=0$. If $F(t)$ happens to be one-valued in $a b$, it is an ordinary continuous function; if $G(t)=F(t)$ at points where the latter is one-valued and at other points $G(t)$ has one of the values of $F(t)$, then $G(t)$ is a one-valued pointwise discontinuous function. 
By the method of condensation of singularities* it is easy to build up simple irreducible continua which have an everywhere dense set of points of the second genre. The previous theorem therefore applies, although such continua have only one prime part.

We now turn to the converse theorem.

24. THEOREM. Let $x=f(t)$ denote a correspondence of the set $C=\{x\}$ to the closed segment $T=\{t\}$ having the following properties:

(i) To each $t$ corresponds a point or a point set forming a limited continuum.

(ii) No point of $C$ corresponds to more than one point of $T$.

(iii) $f(t)$ is one-valued and continuous at a set of points everywhere dense in $T$.

(iv) At points $t^{\prime}$ where $f(t)$ is many-valued, $f\left(t^{\prime}\right)$ is the aggregate of accumulation of $f(t)$ as $t \rightarrow t^{\prime}$.

Then $C$ is a simple irreducible continuum.

Proof. That $C$ is limited follows from the definition of the correspondence. It is also easy to see that the image in $C$ of a closed set in $T$ is closed, and vice versa.

It will now be shown that the image in $C$ of a sub-continuum of $T$ is a continuum, and vice versa. Let $F$ be a sub-continuum of $T$ and let $G$ be its image in $C$. If $G$ is not a continuum, there is a partition $G=G_{1}+G_{2}$, where both sets are closed and $G_{1} \cdot G_{2}=0$. Suppose that $F_{1}$ and $F_{2}$ are the images in $T$ of $G_{1}$ and $G_{2}$ respectively. $F_{1}$ and $F_{2}$ are closed. They have no common point $t$, for the image of $t$ is a continuum or a point and hence must lie wholly in $G_{1}$ or $G_{2}$. Thus $F_{1} \cdot F_{2}=0$, which contradicts the hypothesis that $F$ is a continuum. Hence $G$ is a continuum.

Conversely, let $G$ be a sub-continuum of $C$ and let $F$ be the subset of $T$ to which correspond $\dagger$ points in $G$. The set $F$ is closed. If $F$ is not a continuum, we have $F=F_{1}+F_{2}, F_{1} \cdot F_{2}=0$, and $F_{1}$ and $F_{2}$ are closed. Then $G=G_{1}+G_{2}$, where $G_{1}$ and $G_{2}$ are closed and $G_{1} \cdot G_{2}=0$, contrary to the hypothesis. Hence, if $G$ is a continuum, so is $F$.

It will now be shown that, if the image of a point $t_{0}$ of $T$ is a single point $x_{0}$, then $x_{0}$ is a point of the first genre. For every $\epsilon>0$ there is a $\delta>0$ such that $x$ is a point of $V_{a}\left(x_{0}\right)$ for every $t$ in $V_{\delta}\left(t_{0}\right)$. Let $t^{\prime}<t_{0}$ and $t^{\prime \prime}>t_{0}$ be points in $V_{\boldsymbol{z}}\left(t_{0}\right)$ whose images are single points. Then the image in $C$ of $t^{\prime} t^{\prime \prime}$ is a continuum contained wholly in $V_{e}\left(x_{0}\right)$ while the images of the intervals

\footnotetext{
- See E. W. Hobson, Theory of Functions of a Real Variable, p. 618.

$\dagger$ It should be noted that in general $G$ will be only a part of the set of points of $C$ corresponding to points of $F$.
} 
$0, t^{\prime}$ and $t^{\prime \prime}, 1$ are continua neither of which contains $x_{0}$. Hence for $\eta>0$ sufficiently small, $V_{\eta}\left(x_{0}\right)$ is contained in the image of $t^{\prime} t^{\prime \prime}$. Thus for any $\epsilon>0$ there is an $\eta>0$ such that there is a sub-continuum of $C$ lying in $V_{a}\left(x_{0}\right)$ and containing $V_{\eta}\left(x_{0}\right)$. Therefore $\tau\left(x_{0}\right)=0$.

Since the points of $C$ are either unique images of points of $T$ or are limiting points of this set, this shows that the points of the first genre are everywhere dense in $C$.

Now let $a=f(0)$ and $b=f(1)$. If $D$ is a sub-continuum of $C$ which contains $a$ and $b$, its image in $T$ must be $T$ itself since it is a continuum containing the points 0 and 1 . Hence $D$ must contain all the points of the first genre of $C$. As these are everywhere dense in $C, D=C$. Hence $C$ is irreducible between $a$ and $b$.

This completes the proof of the theorem.

YaLe UNIVERSITY, New Haven, Conn. 